\title{
Prof. Hecheng Li: focusing on clinical research to provide more data support for robotic surgery
}

Submitted Nov 25, 2018. Accepted for publication Dec 06, 2018.

doi: $10.21037 /$ jtd.2018.12.25

View this article at: http://dx.doi.org/10.21037/jtd.2018.12.25

\section{Editor's note}

The 3rd Ruijin International Thoracic Surgery Symposium was successfully held in Shanghai from 15th to 16th of November in 2018, gathering many renowned experts and scholars from the frontier field at home and abroad to discuss the latest progress and hot issues in thoracic surgery.

During the meeting, we were honored to have Professor Hecheng Li, from Ruijin Hospital, Shanghai Jiaotong University, to have an interview.

\section{Expert introduction}

Hecheng Li (Figure 1), MD, Department of Thoracic Surgery, Ruijin Hospital, Shanghai, China.

Prof. Hecheng Li is the Chair of Department of Thoracic Surgery, Ruijin Hospital, Shanghai Jiaotong University, School of Medicine.

He obtained MD in Fudan University Shanghai Cancer Center, then worked there as attending physician. In 2004 and 2011, he became Clinical Research Fellow at Vanderbilt-Ingram Cancer Center, Duke University Medical Center and University of Texas MD Anderson Cancer Center. Since 2014, Prof. Li has become the Chair of Department of Thoracic Surgery, Ruijin Hospital.

He is Chairman of the youth group of Shanghai Anticancer Association professional committee of thoracic oncology. He also holds the membership of Society of Thoracic Surgeons, International Association for the Study of Lung Cancer, American Society of Clinical Oncology, American Association for Cancer Research, Chinese Society of Clinical Oncology, International Chinese Society of Thoracic Surgery, Union for International Cancer Control.

$\mathrm{He}$ is familiar with the comprehensive treatment of lung cancer, esophageal cancer, cardia cancer and mediastinal tumor based on surgery, and skilled in minimally invasive surgery for lung cancer and esophageal cancer. In recent years, he focuses on da Vinci Surgical Robot assisted surgery for thoracic neoplasms.

\section{Interview questions}

(I) Compared with traditional open surgery, what are the advantages of robotic surgery in the $\mathrm{R} 0$ resection?

(II) We learned that Ruijin Hospital has achieved a lot last year. Would you like to share with us breakthroughs made in 2018?

(III) What are the necessities on perioperative management of the prevention of complications in thoracic surgery? What role can technology play?

(IV) Would you like to share Ruijin Hospital's experience in perioperative management of the prevention of thoracic complications, especially in lung protection?

(V) As we know, the Department of Thoracic Surgery of Ruijin Hospital has established the enhanced recovery system in 2015. What benefits the system has brought to patients?

\section{Interview summary}

Shanghai Ruijin Hospital is one of the earliest medical centers in China able to operate segmentectomy, accumulating rich experience in robotic surgery. The new book Robotic Thoracic Surgery: Ruijin Hospital Experience, summarizing the successful robotic operations completed by Ruijin Hospital, was released in this conference (Figure 2). As the Chair of Department of Thoracic Surgery, Prof. Li told us that robotic surgery can excel at the following aspects: first of all, robots can provide doctors with more detailed 3-D view of the operating site than the human eye can do, making the boundaries between normal and abnormal tissues clearer. In addition, plus other auxiliary means, such as ICG molecular fluorescence imaging technology, the edge of the tumor can be more accurate to be determined. Moreover, the special robotic arms can operate even in a very tiny space, giving the surgeon unprecedented control in a minimally invasive environment to achieve a more stable, accurate, all-round operation and achieve $\mathrm{R} 0$ resection. 


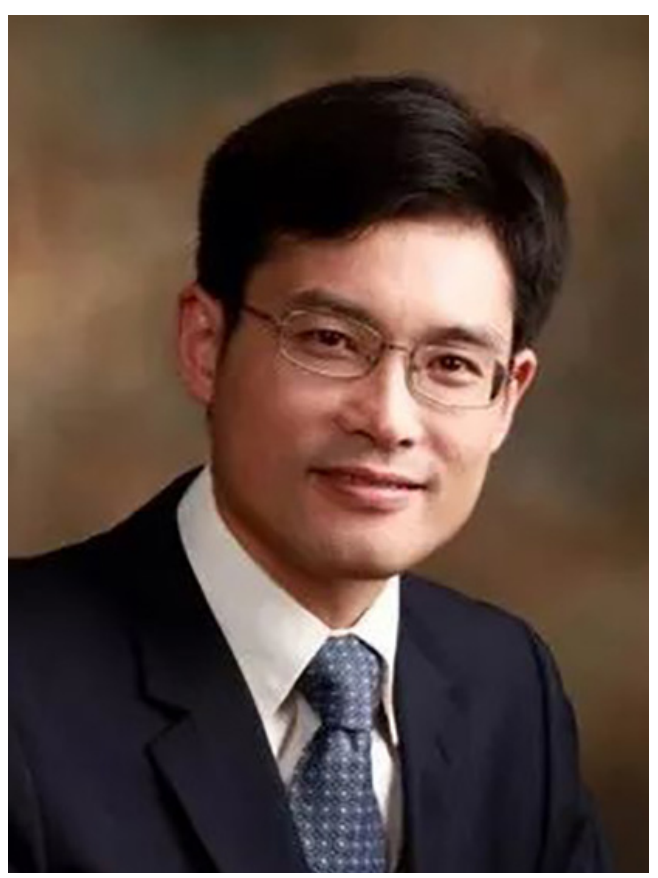

Figure 1 Professor Hecheng Li.

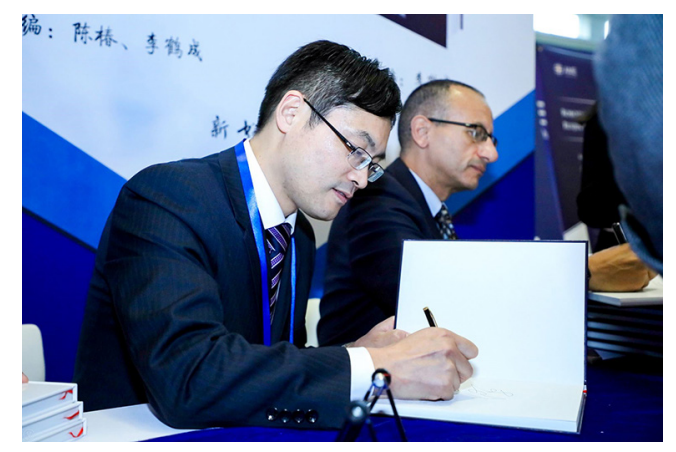

Figure 2 Professor Hecheng Li is signing for the new book Robotic Thoracic Surgery: Ruijin Hospital Experience.

Last year, Ruijin Hospital had done 330 cases of robot surgery, ranked second in China. Furthermore, it is also the first one to successfully operate Ivor Lewis esophagectomy in China. The book Robotic Thoracic Surgery: Ruijin Hospital Experience, therefore, comes at a prime time for the discipline, and it will likely become a useful guide for thoracic surgeons in China and internationally.

Prof. Li said that compared with what had achieved in 2017, in this year, he and his team are putting much more efforts on prospective study to provide more solid data support for robotic surgery with the goal of better applying of robotic surgery, video-assisted thoracoscopic surgery (VATS) so that patients can get the best treatment.

As for how to prevent the incidence of complications in thoracic surgery, Prof. Li said that there are three important factors deserved particular attention. First, surgeons have to study the patients well and do reasonable selection in preoperative preparation. Secondly, surgeons should do their best in realizing less trauma on the patient's body and faster recovery for patients. In this regard, with the progress of science and technology, including the emergence of Leonardo da Vinci robots, it can be greatly improved. Last but not least, postoperative management is cannot be ignored.

Surgery is an important part of the whole disease treatment. But patient's rapid recovery after surgery is also of great significance. Therefore, the Department of Thoracic Surgery of Ruijin Hospital established the enhanced recovery system in 2015.. Prof. Li told us that although the number of patients in thoracic surgery and the proportion of difficult surgeries continue unabated, the patient's hospitalization time does not prolong but shortened because of the good implementation of the system. In the future, efforts will continue to be made in perfecting the system to provide patients with better treatment options.

\section{Acknowledgements}

The author would like to thank Prof. Hecheng Li for participating in the interview.

\section{Footnote}

Conflicts of Interest: The author has no conflicts of interest to declare.

(Science Editor: Amber Yuan, JTD, jtd@amepc.org)
Cite this article as: Yuan A. Prof. Hecheng Li: focusing on clinical research to provide more data support for robotic surgery. J Thorac Dis 2018;10(12):E848-E849. doi: 10.21037/ jtd.2018.12.25 